\title{
L'utilisation des nouvelles technologies éducatives en formation médicale initiale Une enquête à la faculté de médecine et de pharmacie de Casablanca
}

\author{
The use of new educational technologies in medical education \\ A survey at Casablanca University Medical School
}

Internet est un outil de communication de masse incontournable. Au-delà de son usage personnel et citoyen, il permet la mise à disposition de multiples ressources pour l'enseignement et l'apprentissage en formation initiale ou continue. Leur usage doit être pensé dans le cadre plus large des technologies de l'information et de la communication éducatives (TICE). Sous certaines conditions, l'implantation de ces TICE dans les curriculums de formation des professionnels de la santé peut apporter une valeur ajoutée didactique et pédagogique. Elles peuvent notamment faciliter l'accès aux ressources scientifiques, favoriser le travail individuel et offrir une interface permettant de soutenir de multiples interactions entre les formateurs et les étudiants, ou entre les étudiants, par exemple dans le cadre de travaux de groupe collaboratifs.

L'accès à Internet reste cependant inégal. Ainsi, dès 2003, $81 \%$ des établissements d'enseignement supérieur aux Etats-Unis étaient dotés d'un site Internet à visée pédagogique ${ }^{[1]}$. Même si d'importants efforts ont été consentis par de nombreux états, à l'instar de ceux déployés au Maroc par le ministère de l'enseignement supérieur pour favoriser l'achat des ordinateurs personnels au profit des étudiants, ainsi que leur accès à des connexions à haut débit, la situation reste moins favorable dans de nombreux pays. Selon un rapport publié en 2012 par le World Economic Forum (The Global Information Technology Report), le Maroc est positionné en $91^{\mathrm{ème}}$ rang, tandis que la Tunisie et l'Algérie pointent respectivement en $50^{\text {ème }}$ et en $118^{\text {ème }}$ positions ${ }^{[2]}$.

Parallèlement, beaucoup de pays s'orientent vers des dispositifs curriculaires mixtes, recourant à la fois à la formation présentielle sur campus et à l'enseignement à distance. Au Maroc, compte tenu du nombre important d'étudiants à former en médecine, l'enseignement à distance s'impose ainsi comme l'une des solutions à privilégier. Dans ce cadre, la faculté de médecine et de pharmacie de Casablanca a mis en place une plateforme de eLearning via son programme MedLearn pour ses étudiants. En conséquence, il serait nécessaire que tant les enseignants que les étudiants maîtrisent l'usage des TICE.

Dans ce contexte, nous avons cherché à documenter la fréquence et les modalités d'utilisation d'Internet et des TICE par les étudiants de la faculté 
de médecine et de pharmacie de Casablanca dans le cadre de leurs activités académiques. Une enquête prospective, transversale, a ainsi été menée durant deux mois, entre le $1^{\text {er }}$ Mai 2015 et le 30 Juin 2015 , au niveau du centre hospitalo-universitaire (CHU) Ibn Rochd.

Un questionnaire relatif à l'utilisation d'Internet et du e-Learning par les étudiants en médecine, préalablement testé auprès de cinq étudiants, a été administré à tous les étudiants de troisième et quatrième années des études médicales, en stage dans les services de biologie, de pneumologie, d'infectiologie et de médecine interne. Les réponses de 70 étudiants, parmi lesquels 53 femmes $(75,7 \%)$ et 17 hommes $(24,3 \%)$ ont pu être exploitées.

Tous les étudiants, sauf un, ont déclaré posséder un ordinateur portable personnel et 95,7 \% avaient un accès Internet chez eux. La presque totalité d'entre eux $(95,7 \%)$ utilisaient cet outil dans le cadre de leurs études, avec une moyenne de durée de connexion d'environ une heure par semaine mais de larges variations individuelles (68 minutes ; extrêmes : cinq et 320 minutes), sans différence selon le genre et l'année d'étude. Plusieurs raisons étaient invoquées par les étudiants pour rendre compte de cette faible utilisation d'internet dans le cadre de leurs études : manque d'information concernant les sites à consulter $(41,4 \%)$, mauvaise qualité de connexion $(4,3 \%)$, ou insuffisance des ressources mises en ligne $(1,4 \%)$, $52,9 \%$ des étudiants n'ayant pas précisé le motif.

Les sites les plus fréquentés par les étudiants en médecine dans le cadre de leurs études sont les groupes Facebook, Wikipédia, YouTube et les sites des facultés françaises ; 97,1\% des étudiants ne connaissent pas les MOOCS, 98,6\% ne connaissent pas le e-Learning et aucun étudiant ne connait le programme MedLearn.

L'orientation vers un recours accru aux TICE dans le cadre des programmes de formation médicale est affirmée par les universités marocaines depuis plusieurs années. L'université Cadi Ayyad de Marrakech a procédé, depuis maintenant une dizaine d'années à la mise en place d'une plateforme Moodle que les enseignants chercheurs de l'université alimentent régulièrement en contenus numériques de leurs cours, travaux dirigés et travaux pratiques ${ }^{[3]}$.
Elle propose également une plateforme d'accès aux MOOCs avec le projet Uc@Mooc, qui permet aux étudiants d'accéder à des cours, sous forme de vidéos via Internet. L'université Mohammed V Souissi de Rabat a de son côté mis en place un centre de e-Learning et une plateforme Moodle de cours en ligne.

Ces différents programmes sont ambitieux. Le projet MedLearn, mis en place à la faculté de médecine et de pharmacie de Casablanca, a ainsi impliqué 32 personnes, parmi lesquelles 17 professeurs, un médecin spécialiste, six résidents, deux techniciens ainsi qu'une assistante pédagogique. Le personnel concerné a bénéficié de plusieurs formations.

Au regard de ces différents investissements, les résultats de notre enquête confirment l'impact positif des efforts déployés par le ministère de l'enseignement supérieur en collaboration avec les sociétés de télécommunications via le programme INJAZ. puisque quasiment tous les étudiants, quelle que soit leur classe sociale d' origine, possèdent un ordinateur personnel et ont un accès Internet chez eux, même s'ils sont issus de classes sociales différentes.

En revanche, elle confirme un usage très nettement sous optimal des TICE dans le cadre des activités de formation, dans la mesure où une majorité des étudiants recourent essentiellement aux ressources communes (par exemple, Wikipédia ou Google) plutôt qu'aux ressources académiques spécifiques mises à leur disposition. Une très large majorité des étudiants méconnait ainsi les MOOCs ou les autres ressources du E-learning auxquelles ils pourraient avoir accès.

Les étudiants utilisent aussi les réseaux sociaux comme les groupes Facebook, pour le partage des ressources didactiques liées aux cours (fichiers textes ou diaporamas) et comme moyen d'accès à des forums de discussion. De telles interactions sont potentiellement intéressantes mais, en l'absence de supervision par des enseignants, la qualité de ces interactions, à la fois au regard des ressources échangées et des stratégies d'études qu'elles permettent, reste spéculative.

En conclusion, les résultats de notre enquête montrent que la seule implantation, même volontariste, de programmes visant à donner accès aux étudiants aux supports logistiques des TICE ne suffit pas 
à en garantir un usage pédagogique optimal. Si l'on souhaite que toutes les promesses du recours aux TICE en formation médicale soient tenues, au regard de leur formidable potentiel pour faire face aux contraintes et aux problèmes qu'ont à affronter les pays tels que ceux du Maghreb, il importe que toutes les parties prenantes prennent conscience que l'implantation de la logisitique permettant d'accéder à ces ressources doit impérativement s'accompagner de programmes adéquats, vivant à la fois à informer les étudiants de la disponiblité de ces ressources mais aussi à les outiller des compétences nécessaires pour qu'ils les utilisent, en interactions avec leurs enseignants et avec leurs pairs. Il importe aussi, probablement, que les currculums soient revus en profondeur pour tenir compte des objectifs visés, afin d'éviter que les TICE ne soient pas simplement des ressources additionnelles facultatives, plaquées sur des dispositifs demeurant par ailleurs très conventionnels, majoritairement centrés sur des approches transmissives dans une logique axée sur l'enseignement, plutôt que sur des approches interactives dans une logique axée sur l'apprentissage.

\section{Référence}

1. Allen IESJ. Sizing the opportunity: The quality and extent of online education in the United States, 2002-2003. Needham (MA): The Sloan Consortium, Sloan Center for Online Education, 2003.

2. Dutta S, Bilbao-Osorio B. The Global Information Technology Report 2012. Living in a Hyperconnected World. World Economic Forum and INSEAD. 2012 [On-line] Disponible sur : http:// www3.weforum.org/docs/ Global_IT_Report_2012.pdf

3. E-learning quellesavancées pour le Maroc ? Revue de l'étudiant marocain. 2013 / $2014\left(\mathrm{n}^{\circ} 108\right)$ : 2-5. 2014 [On-line] Disponible sur : http://www.rekrute.com/ files-press-media/e-lerning_2014.pdf

Aissam EL Maataoui, Université Ibn Zohr, Faculté de médecine et de pharmacie d'Agadir. Université Hassan II, Faculté de médecine et de pharmacie de Casablanca. BP 32/S Agadir 80000, Maroc

Mailto : a.elmaataoui@uiz.ac.ma

Souad Chaouir, Université Mohamed V, Faculté de médecine et de pharmacie de Rabat. 